\title{
Transarterial Ethanol Ablation Combined with Transarterial Chemoembolization for Hepatocellular Carcinoma with Portal Vein Tumor Thrombus
}

\author{
Biao Yang, ${ }^{1}$ Xin You, ${ }^{1}$ Min lan Yuan, ${ }^{2}$ Tian Qiang Qin, ${ }^{4}$ Lin Jia Duan, ${ }^{3}$ Jiao He, ${ }^{3}$ Ze Jun Fei, ${ }^{3}$ Xuan Zhou, ${ }^{3}$ \\ Rui Yu Zan, ${ }^{1}$ and Zheng Yin Liao ${ }^{1, *}$ \\ ${ }^{1}$ Department of Abdominal Oncology, Cancer Center and State Key Laboratory of Biotherapy, West China Hospital, West China Medical School, Sichuan University, Chengdu, \\ China \\ ${ }^{2}$ Psychiatry Centre, West China Hospital, West China Medical School, Sichuan University, Chengdu, China \\ ${ }^{3}$ Department of Radiology, West China Hospital, West China Medical School, Sichuan University, Chengdu, China \\ ${ }^{4}$ Chinese Evidence-Based Medicine Centre, West China Hospital, West China Medical School, Sichuan University, Chengdu, China \\ "Corresponding author: Zheng Yin Liao, Department of Abdominal Oncology, Cancer Center and State Key Laboratory of Biotherapy, West China Hospital, West China Medical \\ School, Sichuan University, Chengdu, China. Tel: +86-13981717611, E-mail: liaozhengyin@163.com
}

Received 2016 March 04; Revised 2016 May 23; Accepted 2016 June 19.

\begin{abstract}
Background: The prognosis for hepatocellular carcinoma (HCC) with portal vein tumor thrombosis (PVTT) is extremely poor. Objectives: This study aimed to evaluate the safety and effectiveness of transarterial ethanol ablation (TEA) for the treatment of HCC with PVTT.

Methods: Patients were treated with TEA for PVTT under cone-beam computed tomography and traditional transarterial chemoembolization (TACE) with epirubicin for intrahepatic lesions.

Results: Seventeen men were successfully treated with TACE plus TEA. The mean overall survival was $18.3 \pm 9.0$ months (95\% CI: 13.7 -3.0 months). The quality of life (QoL) score increased from $56.9 \pm 15.7$ before the procedure to $88.5 \pm 11.7$ at 4 weeks after the procedure. Lipiodol accumulation grades of 3, 2, 1, and 0 were obtained in $3(17.6 \%), 8$ (47.1\%), 6 (35.3\%), and 0 (0\%) patients, respectively. Conclusions: TEA is a safe and effective method for treating patients with PVTT, offering advantages for QoL, response rate after TEA, and OS.
\end{abstract}

Keywords: Portal Vein Tumor Thrombosis, Ethanol, Transarterial Ethanol Ablation, Hepatocellular Carcinoma, Cone-Beam Computed Tomography

\section{Background}

Hepatocellular carcinoma (HCC) is the fifth most common cancer in the world and the third most prevalent cause of tumor-related death (1). Unfortunately, HCC has a propensity to invade the portal vein, causing portal vein tumor thrombosis (PVTT) (2). Approximately 30.0\% - 62.2\% of patients with HCC develop PVTT (3). The Liver Cancer Study Group of Japan suggested the following four-level classification scheme for PVTT: portal vein invasion class (Vp) 0 , no PVTT; Vp1, existence of PVTT not in, but distal to, secondary portal-vein branches; Vp2, PVTT in secondary portal-vein branches; Vp4, PVTT in primary portal vein branches; and Vp3, PVTT in the main trunk of the portal vein or a portal vein branch contralateral to the main involved lobe (or both) (4). HCC with Vp3 or Vp4 PVTT is an end-stage condition with an extremely poor prognosis because of the risk of tumor cells spreading throughout the liver (5).
Hepatic resection and liver transplantation are the only potentially curative treatments for HCC. However, PVTT is a contraindication for surgery because of the poor prognosis and high surgical risk associated with it(6). Various nonsurgical therapeutic options have been attempted in HCC patients with PVTT, including traditional transarterial chemoembolization (TACE), transarterial embolization, chemotherapy, percutaneous ethanol injection, radiofrequency ablation, and radiation. Unfortunately, none of these treatments is effective (7-9). The median survival of untreated HCC patients with PVTT is 2.7 months (10). Hence, PVTT is an independent risk factor for an extremely poor prognosis in HCC. Although treating PVTT may prolong overall survival (OS) time in HCC patients, there are no effective treatment options for PVTT, especially advanced cases (Vp3/Vp4). Therefore, there is a critical need for an effective treatment strategy for PVTT. 
Transcatheter arterial embolization with a mixture of lipiodol and ethanol has been shown to be an effective treatment for intrahepatic HCC lesions, although it is not widely recognized or used (11-13). The feeding vessels of PVTT are complicated (14). In most PVTT cases ( 92.3\%), blood supply characteristics similar to those of tumors of the intrahepatic portion (TIHPs) are observed. Most of the nutrient vessels of PVTT are liver arteries, with portal veins and/or liver arteries providing supplemental blood supplies.

\section{Objectives}

With the goal of improving median OS, based on this model, we present a technique of traditional TACE for TIHPs using a lipiodol-ethanol mixture (LEM) for transarterial ethanol ablation (TEA) for the treatment of advanced PVTT.

\section{Methods}

\subsection{Patients and Selection Criteria}

This prospective study was approved by the ethics committee of West China hospital cancer center. All patients provided written informed consent for participation. The principles of the World Medical Association Declaration of Helsinki and Good Clinical Practice Guidelines were strictly followed.

Patients were included in this study if they exhibited nonresectable HCC with end-stage PVTT (Vp3/Vp4), adequate coagulation function, and a Child-Pugh classification of $\mathrm{A} / \mathrm{B}$, and they had refused to undergo other therapies and had not received active treatments (e.g., surgery or other conventional/regional treatment) in the previous 6 months. HCC was confirmed by biopsy of a previous liver resection specimen or by satisfying three conditions: 1) the patient tested positive for the hepatitis B virus (HBV), 2) the patient had an abnormal alpha-fetoprotein (AFP) level, and 3 ) the treated lesion showed typical computed tomographic (CT) and angiographic features of HCC. Pregnant patients and children with HCC were excluded due to the uncertain risks of X-ray radiation. Other exclusion criteria were an HBV-DNA value exceeding $1.0 \times 103$ copies/mL, an age of 75 years or older, and an Eastern Cooperative Oncology Group (ECOG) score exceeding 2.

Patients with histologically or cytologically proven HCC were enrolled. Patients with $1+2(1)$ or $1+2(2)+3$ of the following conditions, however, were excluded: 1 . cirrhosis associated with HBV or HCV infection; 2. atypical HCC characteristics on dynamic imaging (i.e., 4-phase multidetector CT/dynamic contrast enhanced magnetic resonance [MR] imaging), with (1) lesions larger than $2 \mathrm{~cm}$ in diameter according to at least one dynamic imaging technique or (2) with lesions 1 - $2 \mathrm{~cm}$ in diameter demonstrated by two dynamic imaging techniques; and 3. An elevated serum AFP level of $>400 \mathrm{ng} / \mathrm{mL}$ for at least one month or $>200 \mathrm{ng} / \mathrm{mL}$ for at least two months, without an alternative explanatory cause.

\subsection{Preoperative Preparation}

Before enrollment, patients provided their basic medical history, underwent a physical examination, and submitted to laboratory testing. The presence of TIHP or PVTT was evaluated by enhanced CT and/or MR imaging. Both oncologists and radiologists assessed the areas at risk for subclinical disease.

\subsection{Treatment Protocol}

In all patients, the Seldinger technique under local anesthesia was used to access the femoral artery. All interventional procedures were performed with the same angiographic system (Allura Xper FD20; Philips Healthcare) under the supervision of at least one of two interventional radiologists, each with more than 20 years' experience in vascular interventions.

The TEA procedures (15) were performed by using a microcatheter with a 2F tip (Progreat $\alpha$, Terumo Clinical Supply, Japan) through a $4 \mathrm{~F}$ catheter to identify the potential PVTT-feeding artery (PFA) under digital subtraction angiography (DSA). Selective cone-beam computed tomography (CBCT) images were used to confirm the PFA. For each CBCT scan (16), 6 - 20 mL of contrast material (Omnipaque, Bracco-Sine, Shanghai, China) was injected within 6-10 seconds under a pressure of 100 - 350 MPa. LEM (1:1 $\mathrm{v} / \mathrm{v}$ ratio of lipiodol to ethanol) was injected through the PFA at a rate of $0.5-1 \mathrm{~mL} / \mathrm{min}$ until blood flow through the PVTT ceased. Next, gelatin-sponge particles (Gelfoam; Fukangseng, Guilin, China) were crushed into approximately $0.2-0.5-\mathrm{mm}$ particles and injected. Lastly, TIHPs were treated by traditional TACE with a mixture of lipiodol and $5 \mathrm{mg} / \mathrm{mL}$ of epirubicin ( $\leq 70 \mathrm{mg}$; Pfizer, Wuxi, China) and crushed gelatin-sponge particles. TACE was finished when the tumor-feeding branch was completely obstructed and the tumor stain disappeared on DSA. To decrease the risk of puncture-point thrombosis, the catheter was filled with undiluted heparin $(1000 \mathrm{U} / \mathrm{mL})$ before it was removed from the femoral artery.

\subsection{Follow-Up and Assessment Indices}

The primary outcomes of the study were lipiodol retention by the PVTT and OS after the intervention. Secondary 
outcomes included quality of life (QoL) scores, the objective response (OR) of the TIHPs, and adverse events. The CT and MR images were assessed by two radiologists.

\subsection{Evaluation of embolization efficacy in PVTT}

To describe the embolization efficiency, lipiodol retention was defined as the ratio of the volume of lipiodolstained tumor tissue 1 week after treatment (Figure1 and 2). Four grades of lipiodol accumulation were defined: grade 3 , complete accumulation in the tumor; grade 2, partial accumulation ( $\geq 50 \%$ of PVTT volume) in the tumor; grade 1 , poor accumulation ( $<50 \%$ of PVTT volume) in the tumor; and grade 0 , no accumulation in the tumor. Lipiodol retention was achieved when the lipiodol accumulation grade was grade 3,2 , or 1 . CT data on the volume of lipiodolstained tumor tissue were obtained by an independent radiologist. Because the tissue organization of postoperative residual thrombi without viability can persist for months or even years, it is difficult to obtain precise statistical descriptions of portal vein recanalization rates.

A 48-year-old man with PVTT (Vp3) was treated by the first procedure and a good lipiodol deposition was obtained. High-density spots representing lipiodol or contrast material were observed. (A, B) A preoperative CT scan was performed showing a large bloc in the portal vein. $(C)$ Potential PFA identified on DSA by abdominal aortagraphy. (D) A microcatheter used under DSA revealed the PFA. (E) Further confirmation of the PFA by enhanced C-arm CT. The high-density region represents the contrast material through the PFA. (F) The post-procedure C-arm CT revealed LEM deposition within the PVTT. (G-I) Axial CT indicating good lipiodol deposition in the PVTT.

\subsection{Evaluation of Treatment Effectiveness in TIHPS}

TIHP response was evaluated based on the RECIST guidelines (version 1.1) (17) using the 3-month CT, whichever was earlier. Tumor responses were classified into 4 categories: complete response (CR), disappearance of all target lesions; partial response (PR), lesion diameter reduction by $30 \%$; stable disease (StD), neither sufficient shrinkage to qualify for PR nor sufficient increase to qualify for progressive disease (PrD); and PrD, lesion diameter increased by $20 \%$. The duration of OR was determined for patients with CR plus PR. QoL was assessed by the European Organization for Research and Treatment of Cancer Quality of Life Group questionnaire (EORTC QLQ-C30). QoL assessment, laboratory tests, and CT scans were repeated at 4-week intervals after the first session. The OS time was defined as the duration from the date of the first treatment to the date of death or loss to follow-up.

\subsection{Statistical Analyses}

Data are presented as percentages of patients or the means \pm standard deviations (SDs). In some cases, 95\% confidence intervals (CIs) are provided. Survival curves were estimated by the Kaplan-Meier method. Other results were analyzed by paired tests with the SPSS statistical software, version 20.0 (SPSS Inc., Chicago, IL, USA). Differences with a $\mathrm{P}<0.05$ were considered statistically significant.

\section{Results}

\subsection{Patient Outcomes}

No patient was lost during the follow-up period. The demographic data of all patients are shown in Table 1. Clinical outcomes before, during, and after the procedure are reported in Tables 1 and 2, Appendix 1 in the supplementary file. A total of 17 men, all of whom tested positive for HBV, were included in this study. The diagnosis was demonstrated histologically in 6/17 patients (35.3\%). Three patients had undergone a single surgical resection, two patients had undergone two TACE procedures, and one patient had received a single course of gamma-radiation therapy before the procedure. Two patients had distant metastases in the lungs before the procedure. The average age was $52 \pm 13$ years (range: 28 - 71 years). Thirteen patients had a Child-Pugh classification grade of $\mathrm{A}$, and 4 had a grade of $B$. The average diameter of the largest lesion in each patient was $10.5 \pm 3.0 \mathrm{~cm}$ (range: $4.6-14.8 \mathrm{~cm}$ ). The average total procedure time was $2.0 \pm 0.6$ hours (95\% CI:1.7 - 2.3 hours). The average radiography time of PFA under CBCT was $8.0 \pm$ 2.1 seconds (95\% CI: 6.9 - 9.0 seconds). The average radiography pressure was $247.1 \pm 94.3 \mathrm{bp}$ (95\% CI:198.6-295.6 bp), and the average radiography speed was $1.6 \pm 0.5 \mathrm{~mL} / \mathrm{s}$ (95\% CI:1.3 - $1.8 \mathrm{~mL} / \mathrm{s})$. Average LEM volume administered per patient was $7.5 \pm 4.4 \mathrm{~mL}$ (95\% CI: 5.3-9.8 mL). All patients had at least 3 lesions.

4.2. OS

The median OS time was 22.1 months (mean: $18.1 \pm 9.0$ months, 95\% CI: 9.7-26.4 months). Estimated OS rates at 12, 24 , and 36 months were $81.4 \%, 46.3 \%$, and $11.6 \%$, respectively (Figure 2). The QoL score increased from $56.86 \pm 15.66$ before the procedure to $88.54 \pm 11.73$ at 4 weeks after the procedure $(\mathrm{P}<0.001)$ (Table 3$)$. Social function and episodes of diarrhea and fatigue also improved.

\subsection{Tumor Response}

On the basis of the radiologic criteria for TIHPs, CR, PR, StD, and PrD were achieved after 3 months in 1 (5.9\%), 4 (23.5\%), 9 (52.9\%), and 3 (17.7\%) patients, respectively. Using the CT results obtained 1 week after the first TEA for PVTT, 

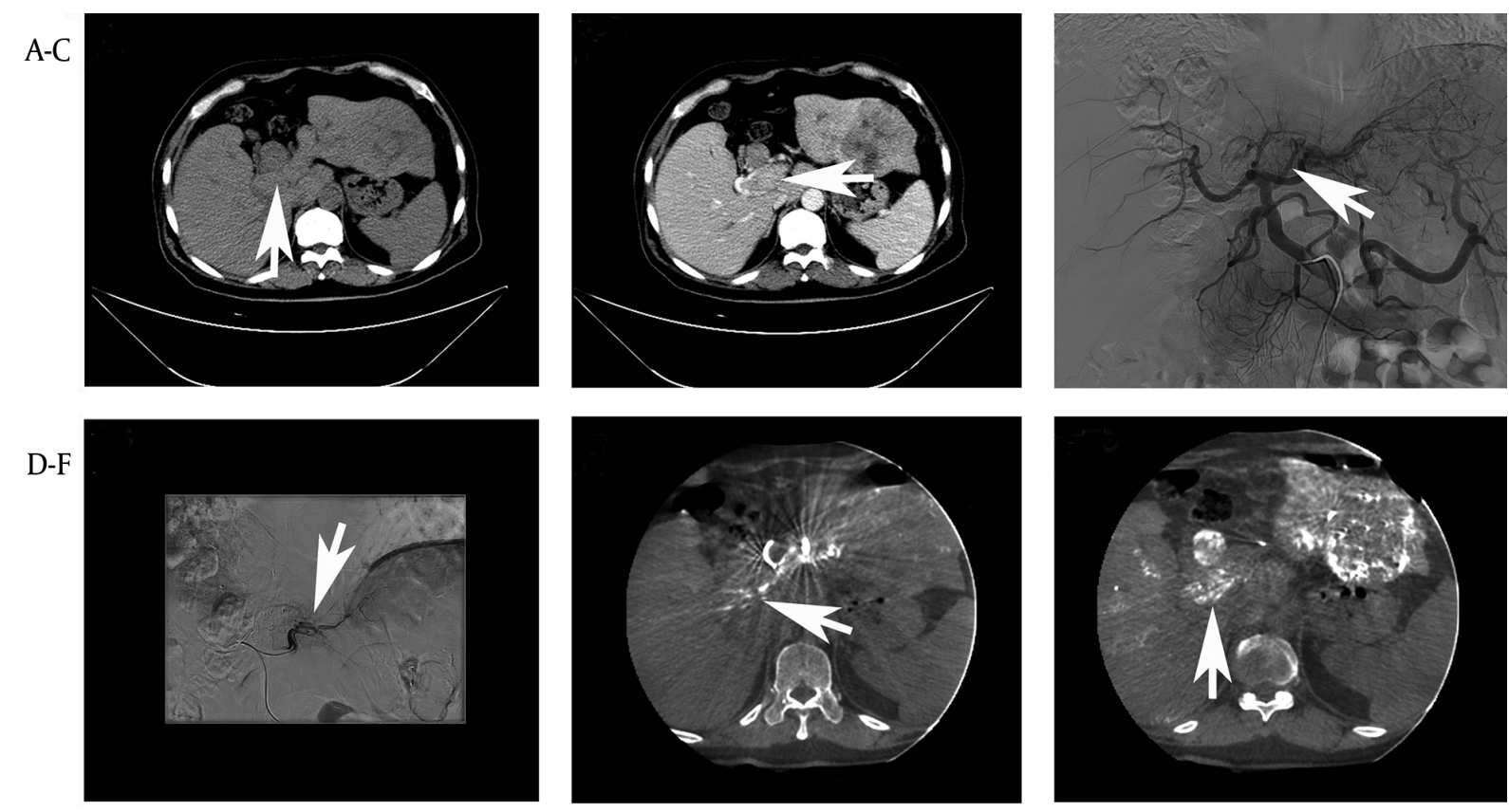

G-1
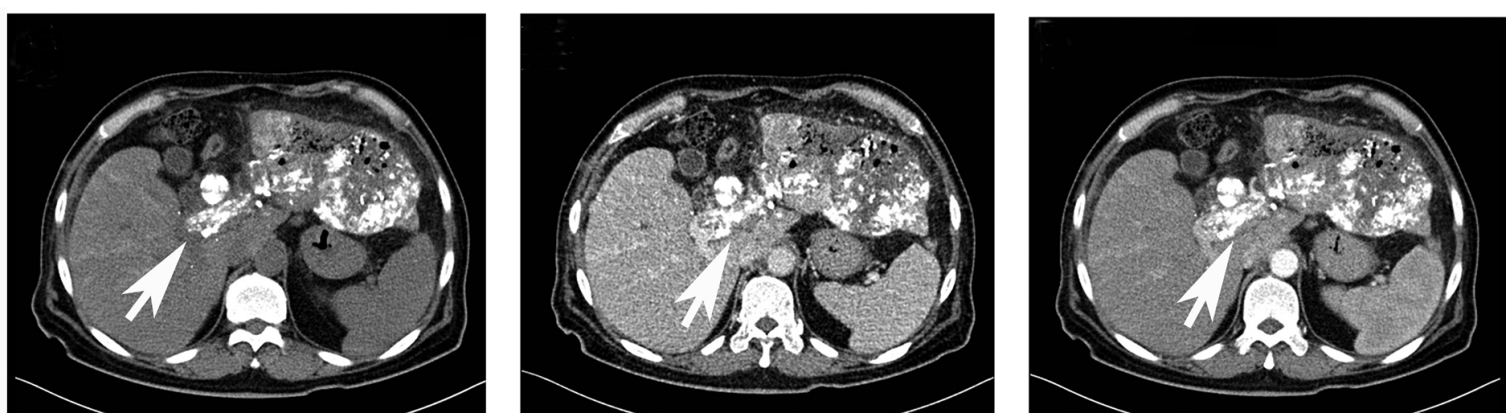

Figure 1. TEA With TACE in 61-Year-Old Male With Unrespectable HCC and PVTT (Vp3)

we found that 3/17 (17.6\%), 8/17 (47.1\%), 6/17 (35.3\%), and $0 / 17$ (0\%) patients achieved lipiodol accumulation grades of 3 , 2,1 , and 0 , respectively.

\subsection{Safety}

No treatment-related deaths occurred. Adverse events are listed in Appendix 2 in the supplementary file. All patients experienced pain after the procedure, with posttreatment fever in 11 patients (64.7\%). Other adverse events after the procedure included vomiting $(n=5)$, gastrointestinal hemorrhage $(n=1)$, ascites $(n=1)$, back pain $(n=$ $7)$, intraoperative vomiting $(n=1)$, epistaxis $(n=1)$, loss of appetite $(n=2)$, thoracalgia $(n=1)$, and fatigue $(n=3)$. After various treatments were performed, all patients recovered within 2 - 15 days. No cases of pulmonary embolism, cholecystitis, cholangitis, or hepatapostema were observed during the follow-up period.

\section{Discussion}

In our patients, TACE was used initially as an adjuvant treatment, but was only helpful for the intraparenchymal portion of the residual tumor. TEA was subsequently used for the tumor thrombus. Our findings indicate important improvements in the management of patients with HCC and end-stage PVTT (Vp3/Vp4). Good retention of transarterially delivered lipiodol was achieved, and the OS percentages at 1 and 2 years (81.4\% and 46.3\%, respectively) were higher than those obtained with TACE alone $30.9 \%$ and 9.2\%, respectively) (18). Kondo et al. (4) found that CR, PR, and StD were achieved in 0/60 (0\%), 8/60 (13.3\%), and 19/60 (31.7\%) of HCC patients with PVTT (Vp2/3/4) who were treated with sorafenib ( $n=60$; median survival time = 5.5 months). In another study of HCC patients with PVTT, after accounting for propensity score, statistically similar 
Table 1. Preoperative Demographic and Clinical Data for Patients With HCC and PVTT ${ }^{a}$

\begin{tabular}{|c|c|}
\hline Variable & Value \\
\hline Male sex & $17(100)$ \\
\hline Age, years & $52 \pm 13(28-71)$ \\
\hline \multicolumn{2}{|l|}{ ECOG status } \\
\hline 0 & $9(52.9)$ \\
\hline 1 & $6(35.3)$ \\
\hline 2 & $2(11.8)$ \\
\hline \multicolumn{2}{|l|}{ Vp classification } \\
\hline Vp3 & $4(23.5)$ \\
\hline Vp4 & $13(76.5)$ \\
\hline Presence of ascites & $4(23.5)$ \\
\hline Presence of cirrhosis & $14(82.4)$ \\
\hline \multicolumn{2}{|l|}{ Child-Pugh score } \\
\hline A & $13(76.5)$ \\
\hline $\mathrm{B}$ & $4(23.5)$ \\
\hline Presence of $\mathrm{HBV} / \mathrm{HCV}$ & $17(100)$ \\
\hline Distant metastasis & $2(11.8)$ \\
\hline $\operatorname{Albumin}(\mathrm{g} / \mathrm{dL})$ & $38.8 \pm 8.9(29.8-69.2)$ \\
\hline Total bilirubin $(\mu \mathrm{mol} / \mathrm{L})$ & $41.8 \pm 83.1(9.2-362.0)$ \\
\hline $\operatorname{ALT}(\mu / \mathrm{L})$ & $67.6 \pm 27.4(19.0-130.0)$ \\
\hline $\operatorname{AST}(\mu / \mathrm{L})$ & $93.5 \pm 55.3(48.0-264.0)$ \\
\hline$\alpha$-Fetoprotein $(\mathrm{ng} / \mathrm{mL})$ & $861.0 \pm 523.0(11.3-1210)^{b}$ \\
\hline
\end{tabular}

Abbreviations: HBV, hepatitis B virus; HCV, hepatitis C virus; Vp, PVTT level; ALT, alanine aminotransferase; AST, aspartate aminotransferase.

${ }^{a}$ Data are reported as the mean \pm standard deviation (range) for continuous variables or as No. (\%) for categorical variables.

${ }^{\mathrm{b}}$ Data exceeding the maximum value of $1210 \mathrm{ng} / \mathrm{mL}$ were considered to be $1210 \mathrm{ng} / \mathrm{mL}$ in the statistics.

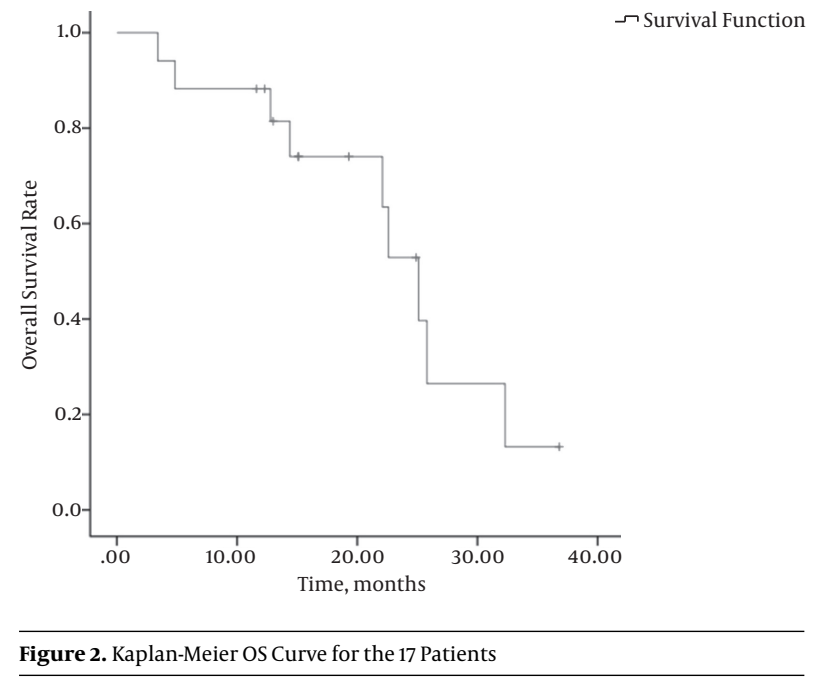

Figure 2. Kaplan-Meier OS Curve for the 17 Patients median survival times were observed in patients treated with sorafenib ( 4.8 months; $\mathrm{n}=40$ ) versus those treated with radiation therapy (5.9 months; $\mathrm{n}=57$ ). These results confirm our hypothesis that treatment of PVTT may prolong OS for patients with HCC and PVTT. We observed some adverse events related to the use of LEM for PVTT treatment. These side effects may be explained by the pain related to the thermogenesis of ethanol. Although some patients exhibited a decreased PVTT diameter after transarterial embolization, they did not undergo recanalization. The reduced diameter can be due to residual, nonviable thrombi that may exist for months or years due to tissue organization (19). On a patient basis, the best tumor response and the disease progression in our study did not add up to $100 \%$ because these outcomes were observed within the study period rather than at a particular time. As these outcomes might have occurred at different times during the study, the selection of outcomes at any particular time might be misleading. Hence, we did not account 
Table 2. Intra- and Postoperative data for Patients With HCC and PVTT ${ }^{\mathrm{a}}$

\begin{tabular}{|c|c|c|}
\hline Variable & Value & $\mathbf{P}$ \\
\hline LIHL, cm & $10.5 \pm 3.0(4.6-14.8)$ & \\
\hline Operation time, h & $2.0 \pm 0.6(1.0-3.0)$ & \\
\hline \multicolumn{3}{|l|}{ Radiography for PVTT } \\
\hline Time, s & $8 \pm 2(5-10)$ & \\
\hline Pressure, bp & $247.1 \pm 94.3(100.0-350.0)$ & \\
\hline Speed, $\mathrm{mL} / \mathrm{s}$ & $1.6 \pm 0.5(1.0-2.0)$ & \\
\hline LEM, $\mathbf{m L}$ & $7.5 \pm 4.4(2.0-17.0)$ & \\
\hline Epirubicin, mg & $49.4 \pm 7.5(30.0-70.0)$ & \\
\hline Operation sessions: $2 ; 3 ; 4$ & $9(52.9) ; 4(23.5) ; 4(23.5)$ & \\
\hline Prothrombin time, $s$ & $13.4 \pm 2.0(11.1-18.5)$ & \\
\hline Thrombin time, $s$ & $19.7 \pm 1.6(16.9-23.6)$ & \\
\hline Albumin, $\mathbf{g} / \mathbf{d}$ & $36.3 \pm 10.2(24.5-72.6)$ & 0.04 \\
\hline Total bilirubin, $\mu \mathrm{mol} / \mathbf{L}$ & $51.4 \pm 61.2(13.7-278.0)$ & 0.23 \\
\hline ALT, $\mu / \mathbf{L}$ & $308.0 \pm 339.0(43.0-1071.0)^{b}$ & 0.02 \\
\hline AST, $\mu / \mathbf{L}$ & $370.4 \pm 573.0(37.0-1920.0)^{\mathrm{b}}$ & 0.08 \\
\hline$\alpha$-Fetoprotein, ng/mL & $471.1 \pm 509.7(6.9-1210)^{\mathrm{c}, \mathrm{d}}$ & 0.81 \\
\hline Follow-up (months) & $18.3 \pm 9.0(3.4-36.8)$ & \\
\hline IAGI: 1; 2;3 & $1(5.9) ; 10(58.8) ; 6(35.3)$ & \\
\hline IAGP: 1; 2;3 & $6(35.3) ; 8(47.1) ; 3(17.6)$ & \\
\hline \multicolumn{3}{|l|}{ Outcome } \\
\hline Alive w/disease & $7(50)$ & \\
\hline Died of disease & $9(52.9)$ & \\
\hline Died of GI hemorrhage & $1(5.9)$ & \\
\hline
\end{tabular}

Abbreviations: IAGP, iodized oil accumulation grade of PVTT; IAGI, iodized oil accumulation grade of intrahepatic lesions; LEM, lipiodol-ethanol mixture; LIHL, largest tumor size among intrahepatic lesions; ALT, alanine aminotransferase; AST, aspartate aminotransferase.

${ }^{a}$ Data are expressed as mean \pm standard deviation (range) for continuous variables of No. (\%) for categorical variables; P values were determined by paired $t$ test for preoperative vs. postoperative values.

${ }^{\mathrm{b}}$ Data for patient 11 or patients 14 and 17, respectively, were lost; statistical data were determined for 16 or 15 patients, respectively.

${ }^{c}$ Values exceeding the maximum value of $1210 \mathrm{ng} / \mathrm{mL}$ were counted as $1210 \mathrm{ng} / \mathrm{mL}$ in the statistics.

${ }^{\mathrm{d}}$ Data for patient 11 or patients 14 and 17, respectively, were lost; statistical data were determined for 16 or 15 patients, respectively.

for the recanalization rate. All patients in this study underwent multiple treatments, with the second TEA being performed less than 4 weeks after the first. In our experience, repeated treatment is necessary because it is unlikely that the PFA will be embolized completely with one session of TEA. Gelatin-sponge particles were injected after LEM injection, which can result in rapid flushing of the LEM and prolonged conservation of the PFA, enhancing treatment effectiveness. Arterioportal shunts are often accompanied by PVTT. Gelatin-sponge particles can impel the LEM to flow slowly into the PV, decreasing the risk of ethanol damage to portal vein endothelial cells. It is our view that multiple sessions are strictly necessary for large tumors (diameter $>10 \mathrm{~cm}$ ). Indeed, attempting to embolize a large-diameter tumor with PVTT completely with a single TEA and TACE session could be quite dangerous.

CBCT angiography must be performed to identify the PFA and to embolize the PVTT by LEM. As the blood supply of the PFA is usually complicated, it may be difficult to distinguish the effects of embolization due to lipiodol retention of TACE from the effects of TEA when traditional TACE for TIHP is performed first. TEA has minimal or no adverse effects on liver function, although a mild transient rise in serum transaminase levels may be observed. Although CBCT angiography can help in identifying the PFA, it is still difficult to locate it when TACE is performed first because the contrast material may be confused with lipiodol. Hence, TEA should be performed before TACE. 
Table 3. QoL Scores Before and Four Weeks after Treatment $(\mathrm{n}=17)^{\mathrm{a}}$

\begin{tabular}{|c|c|c|c|}
\hline EORTC & Preoperative & Postoperative & P Value $^{b}$ \\
\hline Physical function & $89.0 \pm 11.3$ & $95.0 \pm 9.3$ & 0.21 \\
\hline Role function & $91.2 \pm 13.3$ & $10.0 \pm 0.0$ & 0.08 \\
\hline Emotional function & $93.6 \pm 39.6$ & $97.9 \pm 23.9$ & 0.24 \\
\hline Cognitive function & $90.2 \pm 11.9$ & $91.6 \pm 78.9$ & 0.76 \\
\hline Social function & $79.4 \pm 19.1$ & $95.8 \pm 7.7$ & 0.03 \\
\hline Quality of life & $56.9 \pm 15.7$ & $88.5 \pm 11.7$ & $<0.001$ \\
\hline Fatigue & $18.3 \pm 13.6$ & $6.9 \pm 10.2$ & 0.05 \\
\hline Nausea \& vomiting & $4.9 \pm 9.8$ & $8.0 \pm 0.0$ & 0.18 \\
\hline Pain & $28.4 \pm 23.4$ & $10.4 \pm 12.4$ & 0.05 \\
\hline Dyspnea & $11.8 \pm 16.4$ & $4.2 \pm 11.8$ & 0.25 \\
\hline Insomnia & $25.5 \pm 25.1$ & $8.3 \pm 15.4$ & 0.09 \\
\hline Appetite loss & $17.7 \pm 31.4$ & $8.0 \pm 0.0$ & 0.13 \\
\hline Constipation & $15.7 \pm 20.8$ & $12.5 \pm 17.3$ & 0.71 \\
\hline Diarrhea & $15.7 \pm 20.8$ & $8.0 \pm 0.0$ & 0.05 \\
\hline Financial difficulties & $33.3 \pm 33.3$ & $20.8 \pm 17.3$ & 0.33 \\
\hline
\end{tabular}

${ }^{\mathrm{a}}$ Value are expressed as mean $\pm \mathrm{SD}$.

${ }^{\mathrm{b}}$ Paired $\mathrm{t}$ test.

\subsection{Ethanol for PVIT}

Among the available embolic materials, we chose to use absolute ethanol, which is a traditional and inexpensive material. Ethanol is a strong protein coagulant that can inactivate tumor cells directly in situ. This material causes vascular endothelial injury and platelet cohesion, resulting in the permanent occlusion of tumor-feeding vessels and tumor infarction without causing collateral circulation (20). However, ethanol is not radioopaque, and its flow and speed are difficult to visualize after it is administered. In contrast, the use of tracing LEM (1:1 v/v ratio of lipiodol to ethanol) is both feasible and safe for embolism of the PVTT (13). Theoretically, the preserved embolization potency of an LEM allows this medium to embolize portal venules effectively as it passes through the peribiliary plexus to the portal venules (21). Effective embolization of portal venules surrounding the tumor blocks off the portal blood supply to the tumor periphery and prevents the infused agent in the tumor vasculature from draining away through the portal venules (12). If this hypothesis is valid, then the embolization efficacy of LEM is likely to be superior to that of ethanol-free lipiodol formulations, such as those used for chemoembolization (12). Moreover, LEM would achieve a complete thrombotic effect with embolism both in the arteries supplying the tumor and in their adjacent parenchymal PVs (11). Incomplete embolization may lead to ischemia and hypoxia, which may stim- ulate factors related to angiogenesis and carcinogenesis, such as vascular endothelial and fibroblast growth factors. These growth factors, in turn, promote collateral circulation and the restoration of the tumor blood supply, leading to tumor proliferation and recurrence. This scenario, while supporting the use of an LEM in the liver to achieve complete embolization, also creates doubt regarding treatments such as transarterial embolization for HCC (22). However, an LEM is not used to achieve tumor ischemia. Rather, when the tumor vasculature is infiltrated with LEM, diffusion of ethanol into the tumor tissue is likely to achieve complete tumor necrosis, as was shown previously (23).

\subsection{Comparison With Other Therapies for PVTT}

There are few treatment options available for PVTT. En bloc resection of the PVTT seems to be a theoretically superior procedure without exposure of the tumor thrombus. However, this treatment is complicated by high morbidity and mortality (24). Thrombectomy is potentially a noncurative resection approach because tumor cells might be exposed in the surgical field despite careful management. Studies have not demonstrated a survival difference among patients with macroscopic PVTT undergoing thrombectomy or en bloc resection (25). Liver transplantation is not used in patients with HCC and major vascular invasion (26). Moreover, systemic chemotherapy for PVTT 
has not yielded satisfactory results, offering a median survival rate of 3.9 months (27).

Although the safety and effectiveness of TACE for these patients have been demonstrated in selected instances, the survival benefit remains unclear. The 3-year survival rate is only $9 \%$, and the median survival is 5.2 months (28). Radiofrequency ablation is a thermal ablation method that is mostly used for small tumors (ideally $<3 \mathrm{~cm}$ ), especially in the liver (29). Chan et al. (30) attempted to use percutaneous ethanol injections to treat PVTT, but did not achieve a satisfactory outcome. They cited several difficulties in using percutaneous ethanol injections for malignant PVTT. First, it was difficult to assess the exact extent of tumor thrombosis once percutaneous ethanol injections had begun. Hence, the severe complication of biliary stricture was observed. Second, the dosage of alcohol for tumor thrombosis was difficult to estimate because the lesion was not round. Thus, unintended alcohol leakage into the arteries is expected.

\subsection{Limitations}

The conclusions of this study are limited by various factors. First, the duration of follow-up was not prolonged. Nevertheless, the number of patients recruited was sufficient to show significant improvements in embolization efficacy and treatment effectiveness compared to untreated patients. Second, our patients were recruited from a single center, and the sample size was relatively small due to the rarity of HCC with PVTT. Third, we did not perform a multiple-arm study that compared TACE combined with TEA to any other treatments.

\subsection{Conclusions}

TEA with an LEM combined with TACE is not a curative approach for HCC with PVTT. Nevertheless, we have shown that this method is safe, feasible, and effective for the treatment of HCC with PVTT (Vp3/Vp4).

\section{Supplementary Material}

Supplementary material(s) is available here.

\section{Footnotes}

Authors' Contribution: Biao Yang and Xin You contributed equally to this work. Biao Yang and Xin You, both first authors, participated in the study design, sample collection, data extraction and analysis, and writing of the manuscript. Rui-yu Zan provided great help in terms of the study design. Lin-jia Duan resolved all discrepancies as an intercessor. Min-lan Yuan assisted in modifying the manuscript. Tian-qiang Qin, a statistician, contributed to the statistical analysis. Jiao He, Ze-jun Fei, and Xuan Zhou, radiologists, provided help in evaluating medical images. Liao Zheng-yin provided support throughout the entire duration of the study.

Funding/Support: This study was sponsored by the national nature science foundation of China (81470141) and Science and Technology Department of Sichuan Province(2014SZ0002-8) to LZY. The funding organizations are public institutions and had no role in the design and conduct of the study; collection, management, and analysis of the data; or preparation, review, and approval of the manuscript.

\section{References}

1. El-Serag HB, Rudolph KL. Hepatocellular carcinoma: epidemiology and molecular carcinogenesis. Gastroenterology. 2007;132(7):2557-76. doi: 10.1053/j.gastro.2007.04.061. [PubMed: 17570226].

2. Llovet JM, Bustamante J, Castells A, Vilana R, Ayuso Mdel C, Sala M, et al. Natural history of untreated nonsurgical hepatocellular carcinoma: rationale for the design and evaluation of therapeutic trials. Hepatology. 1999;29(1):62-7. doi:10.1002/hep.510290145. [PubMed: 9862851].

3. Esnaola NF, Mirza N, Lauwers GY, Ikai I, Regimbeau JM, Belghiti J, et al. Comparison of clinicopathologic characteristics and outcomes after resection in patients with hepatocellular carcinoma treated in the United States, France, and Japan. Ann Surg. 2003;238(5):711-9. doi: 10.1097/01.sla.0000094436.34556.ac. [PubMed:14578734].

4. Kondo K, Chijiiwa K, Kai M, Otani K, Nagaike K, Ohuchida J, et al. Surgical strategy for hepatocellular carcinoma patients with portal vein tumor thrombus based on prognostic factors. J Gastrointest Surg. 2009;13(6):1078-83. doi: 10.1007/s11605-009-0854-2. [PubMed: 19296182].

5. Ban D, Shimada K, Yamamoto Y, Nara S, Esaki M, Sakamoto Y, et al. Efficacy of a hepatectomy and a tumor thrombectomy for hepatocellular carcinoma with tumor thrombus extending to the main portal vein. J Gastrointest Surg. 2009;13(11):1921-8. doi: 10.1007/s11605-009-0998-0. [PubMed: 19727969].

6. Chen JS, Wang Q, Chen XL, Huang XH, Liang LJ, Lei J, et al. Clinicopathologic characteristics and surgical outcomes of hepatocellular carcinoma with portal vein tumor thrombosis. J Surg Res. 2012;175(2):24350. doi:10.1016/j.jss.2011.03.072. [PubMed: 21601221].

7. Takayasu K, Arii S, Ikai I, Omata M, Okita K, Ichida T, et al. Prospective cohort study of transarterial chemoembolization for unresectable hepatocellular carcinoma in 8510 patients. Gastroenterology. 2006;131(2):461-9. doi: 10.1053/j.gastro.2006.05.021. [PubMed: 16890600]

8. Ohkubo T, Yamamoto J, Sugawara Y, Shimada K, Yamasaki S, Makuuchi $\mathrm{M}$, et al. Surgical results for hepatocellular carcinoma with macroscopic portal vein tumor thrombosis. J Am Coll Surg. 2000;191(6):65760. [PubMed: 11129815].

9. Tanaka A, Morimoto T, Yamaoka Y. Implications of surgical treatment for advanced hepatocellular carcinoma with tumor thrombi in the portal vein. Hepatogastroenterology. 1996;43(9):637-43. [PubMed: 8799408].

10. Pawarode A, Voravud N, Sriuranpong V, Kullavanijaya P, Patt YZ. Natural history of untreated primary hepatocellular carcinoma: a retrospective study of 157 patients. Am J Clin Oncol. 1998;21(4):386-91. [PubMed: 9708639]. 
11. Park JH, Han JK, Chung JW, Choi BI, Han MC, Kim YI. Superselective transcatheter arterial embolization with ethanol and iodized oil for hepatocellular carcinoma. J Vasc Interv Radiol. 1993;4(3):333-9.

12. Yu SC, Hui JW, Hui EP, Mo F, Lee PS, Wong J, et al. Embolization efficacy and treatment effectiveness of transarterial therapy for unresectable hepatocellular carcinoma: a case-controlled comparison of transarterial ethanol ablation with lipiodol-ethanol mixture versus transcatheter arterial chemoembolization. J Vasc Interv Radiol. 2009;20(3):352-9. doi: 10.1016/j.jvir.2008.12.407. [PubMed: 19167240].

13. Gu YK, Luo RG, Huang JH, Si Tu QJ, Li XX, Gao F. Transarterial embolization ablation of hepatocellular carcinoma with a lipiodolethanol mixture. World J Gastroenterol. 2010;16(45):5766-72. [PubMed: 21128329].

14. Si Q, Huang SX, TONG W, Qian XL, Lv XP, Huang YL. Clinical study of blood perfusion characteristics in liver cancer and portal vein tumor thrombosis by CEUS and CDUS. Military Med J Southeast China. 2011;13(1):20-2.

15. U SC, Hui JW, Hui EP, Chan SL, Lee KF, Mo F. Unresectable hepatocellular carcinoma: randomized controlled trial of transarterial ethanol ablation versus transcatheter arterial chemoembolization. Radiol. 2014;270(2):607-20.

16. Miyayama S, Yamashiro M, Hashimoto M, Hashimoto N, Ikuno M, Okumura $\mathrm{K}$, et al. Comparison of local control in transcatheter arterial chemoembolization of hepatocellular carcinoma $<1=6 \mathrm{~cm}$ with or without intraprocedural monitoring of the embolized area using cone-beam computed tomography. Cardiovasc Intervent Radiol. 2014;37(2):388-95. doi: 10.1007/s00270-013-0667-2. [PubMed: 23775550].

17. Eisenhauer EA, Therasse P, Bogaerts J, Schwartz LH, Sargent D, Ford $R$, et al. New response evaluation criteria in solid tumours: revised RECIST guideline (version 1.1). Eur J Cancer. 2009;45(2):228-47. doi: 10.1016/j.ejca.2008.10.026. [PubMed: 19097774].

18. Luo J, Guo RP, Lai EC, Zhang YJ, Lau WY, Chen MS, et al. Transarterial chemoembolization for unresectable hepatocellular carcinoma with portal vein tumor thrombosis: a prospective comparative study. Ann Surg Oncol. 2011;18(2):413-20. doi: 10.1245/s10434-010-1321-8. [PubMed: 20839057].

19. Yoon JH, Kim HC, Chung JW, Yoon JH, Jae HJ, Park JH. CT findings of completely regressed hepatocellular carcinoma with main portal vein tumor thrombosis after transcatheter arterial chemoembolization. Korean J Radiol. 2010;11(1):69-74. doi: 10.3348/kjr.2010.11.1.69. [PubMed: 20046497].

20. Kan Z, Wallace S. Transcatheter liver lobar ablation: an experi- mental trial in an animal model. Eur Radiol. 1997;7(7):1071-5. doi: 10.1007/s003300050256. [PubMed: 9265678].

21. Wallace MJ, Murthy R, Kamat PP, Moore T, Rao SH, Ensor J, et al. Impact of C-arm CT on hepatic arterial interventions for hepatic malignancies. J Vasc Interv Radiol. 2007;18(12):1500-7. doi: 10.1016/j.jvir.2007.07.021. [PubMed: 18057284].

22. Mathupala SP, Rempel A, Pedersen PL. Glucose catabolism in cancer cells: identification and characterization of a marked activation response of the type II hexokinase gene to hypoxic conditions. J Biol Chem. 2001;276(46):43407-12. doi: 10.1074/jbc.M108181200. [PubMed: 11557773].

23. Tanaka K, Okazaki H, Nakamura S, Endo O, Inoue S, Takamura Y, et al. Hepatocellular carcinoma: treatment with a combination therapy of transcatheter arterial embolization and percutaneous ethanol injection. Radiology. 1991;179(3):713-7. doi:10.1148/radiology.179.3.1851313. [PubMed: 1851313].

24. Yamaoka Y, Kumada K, Ino K, Takayasu T, Shimahara Y, Mori K, et al. Liver resection for hepatocellular carcinoma (HCC) with direct removal of tumor thrombi in the main portal vein. World J Surg. 1992;16(6):1172-6. [PubMed: 1333683].

25. Le Treut YP, Hardwigsen J, Ananian P, Saisse J, Gregoire E, Richa H, et al. Resection of hepatocellular carcinoma with tumor thrombus in the major vasculature. A European case-control series. J Gastrointest Surg. 2006;10(6):855-62. doi: 10.1016/j.gassur.2005.12.011. [PubMed: 16769542].

26. Mazzaferro V, Regalia E, Doci R, Andreola S, Pulvirenti A, Bozzetti F, et al. Liver transplantation for the treatment of small hepatocellular carcinomas in patients with cirrhosis. N Engl J Med. 1996;334(11):6939. doi:10.1056/NEJM199603143341104. [PubMed: 8594428].

27. Okada S, Okazaki N, Nose H, Yoshimori M, Aoki K. Prognostic factors in patients with hepatocellular carcinoma receiving systemic chemotherapy. Hepatology. 1992;16(1):112-7. [PubMed: 1377657].

28. Minagawa M, Makuuchi M, Takayama T, Ohtomo K. Selection criteria for hepatectomy in patients with hepatocellular carcinoma and portal vein tumor thrombus. Ann Surg. 2001;233(3):379-84. [PubMed: 11224626].

29. Prunoiu V, Cirimbei C, Marincas M, Cirimbei S, Bratucu E. Extending indication for radiofrequency ablation (585-9RFA) in cancer surgery. Chirurgia (Bucur). 2011;106(5):585-9.

30. Chan MK, Kwok PC, Chan SC, Lam TW, Lo KK, Lam CL. Percutaneous ethanol injection as a possible curative treatment for malignant portal vein thrombosis in hepatocellular carcinoma. Cardiovasc Intervent Radiol. 1999;22(4):326-8. [PubMed:10490334]. 